PROCEEDINGS OF THE

AMERICAN MATHEMATICAL SOCIETY

Volume 128 , Number 12, Pages 3647-3654

S 0002-9939(00)05700-2

Article electronically published on June 21, 2000

\title{
NON-CLOSED SUMS OF CLOSED IDEALS IN BANACH ALGEBRAS
}

\author{
P. G. DIXON
}

(Communicated by David R. Larson)

\begin{abstract}
A major difficulty in Banach algebra theory is that sums of closed ideals need not be closed. We survey the known results and present examples showing that they are in most directions the best possible. We also give a new sufficient condition for closure in the uniform algebra setting.
\end{abstract}

\section{INTRODUCTION}

The study of groups and rings is founded on three basic "Isomorphism Theorems" encountered in most undergraduate mathematics courses. Their analogues for Banach algebras can be stated as follows, all mappings mentioned being continuous homomorphisms and $\cong$ denoting bicontinuous isomorphism of algebras.

Theorem 1.1. Let $A, B$ be Banach algebras, $\theta: A \rightarrow B$, and $I, J, K$ closed ideals in $A$ with $K \subseteq J$. Then:

1) if $\theta$ is a surjection, then $\theta(A) \cong A / \operatorname{ker}(\theta)$;

2) if $I+J$ is closed in $A$, then $I /(I \cap J) \cong(I+J) / J$;

3) $A / J \cong(A / K) /(J / K)$.

The proofs are straightforward, but the reader will note that the hypotheses in the first sentence are essentially those of the algebraic theorems, while the extra hypotheses stated in 1) and 2) are needed in order to make the algebraic isomorphisms bicontinuous (using Banach's Isomorphism Theorem). Moreover, these hypotheses are necessary: if $\theta(A) \cong A / \operatorname{ker}(\theta)$, then $\theta$ is a surjection onto some Banach algebra, and if $I /(I \cap J) \cong(I+J) / J$, then $I+J$ is closed in $A$ (Lemma 2.1 below).

It is the existence of these extra hypotheses which frustrates a programme of developing a structure theory for general Banach algebras by simply translating the extensive body of material on rings into the category of Banach algebras and continuous homomorphisms: the sort of programme evident in parts of [4], for example. We frequently come across continuous homomorphisms $\theta: A \rightarrow B$ whose ranges are not closed. Less frequently do we meet pairs of closed ideals $I, J$ for which $I+J$ is not closed, yet this possibility is at least as serious a hindrance to the construction of a good structure theory. The object of this note is to investigate whether these troublesome pairs of ideals really are a common occurrence or just a

Received by the editors March 1, 1999

2000 Mathematics Subject Classification. Primary 46H10; Secondary 46J20, 46J15.

(C)2000 American Mathematical Society 
pathology of degenerate algebras which may be excised by suitable, mild hypotheses. Sadly, our conclusion is the former-non-closed sums can occur in situations which are otherwise almost as pleasant as one could wish.

I should like to thank numerous members of the functional analysis community for helpful conversations on this subject and the referee for pertinent comments.

\section{Positive Results}

For reference, we restate the remark following Theorem 1.1

Lemma 2.1. If $I, J$ are closed subspaces of a Banach space $X$, then the natural map $I /(I \cap J) \rightarrow(I+J) / J$ is a continuous linear bijection. If its inverse is continuous, then $I+J$ is closed in $X$.

Proof. The fact that $I /(I \cap J) \rightarrow(I+J) / J$ is a continuous linear bijection is elementary. If it is bicontinuous, then $(I+J) / J$ is complete, so closed in $X / J$, so $I+J$ is closed in $X$.

There is an elementary positive result that holds in any Banach algebra: if one of the ideals is finite-dimensional or cofinite-dimensional, then the sum is closed.

Theorem 2.2. If $X$ is a Banach space and $F, C, Y$ are closed subspaces of $X$ with $\operatorname{dim} F<\infty$ and $\operatorname{dim} X / C<\infty$, then $F+Y$ and $C+Y$ are closed.

Proof. The algebraic isomorphisms $(F+Y) / Y \cong F /(F \cap Y)$ and $(C+Y) / C \cong$ $Y /(C \cap Y)$ between finite-dimensional spaces are necessarily continuous, so the result follows from Lemma 2.1

Turning now to results specific to ideals, we find the situation in $\mathrm{C}^{*}$-algebras most encouraging.

Theorem 2.3 (3), Corollary 1.8.4). Let $A$ be a $C^{*}$-algebra, $B$ a sub-C*-algebra of $A$ and $I$ a closed two-sided ideal of $A$. Then $B+I$ is a sub- $C^{*}$-algebra of $A$ and the $C^{*}$-algebras $(B+I) / I$ and $B /(B \cap I)$ are canonically isomorphic.

In particular, it follows that if $I, J$ are closed two-sided ideals of $A$, then $I+J$ is closed. Recall that closed two-sided ideals of $\mathrm{C}^{*}$-algebras are automatically selfadjoint ([3], Proposition 1.8.2).

Later, Combes and Perdrizet ([1], Proposition 6.2) showed that the sum of a closed left ideal and a closed right ideal in a $\mathrm{C}^{*}$-algebra is necessarily closed. This was rediscovered by Rudin ([7], Example 4.6, see also [5], Corollary 32.2), and again by Kirchberg ([6], Lemma 4.9 (iv), see also Wassermann [9], Lemma 8.1). In fact, Rudin's result is more general:

Proposition 2.4. Let $L$ be a closed left ideal and $R$ a closed right ideal, in a Banach algebra $A$. If the algebra $L$ has a bounded right approximate identity (or, by symmetry, if $R$ has a bounded left approximate identity), then $L+R$ is closed.

For completeness, we include a proof here, which is Wassermann's proof with the $\mathrm{C}^{*}$-algebra theory removed. Rudin's proof proceeds through a more general result and so is less succinct.

Proof. Let $\left(e_{\lambda}\right) \subseteq L$ be a bounded right approximate identity for $L$, with bound $M$. That is, $a e_{\lambda} \rightarrow a$ for all $a \in L$ and $\left\|e_{\lambda}\right\| \leq M$ for all $\lambda$. By Lemma 2.1, it suffices 
to prove that the natural map $(L+R) / R \rightarrow L /(L \cap R)$ is continuous. For $a \in L$ and any $r \in R$ we have

$$
\begin{aligned}
\|a-r\| & \geq M^{-1}\left\|a e_{\lambda}-r e_{\lambda}\right\| \\
& \geq M^{-1}\left(\left\|a-r e_{\lambda}\right\|-\left\|a-a e_{\lambda}\right\|\right) \\
& \geq M^{-1}\left(\|a+(L \cap R)\|_{L /(L \cap R)}-\left\|a-a e_{\lambda}\right\|\right) \\
& \rightarrow M^{-1}\left(\|a+(L \cap R)\|_{L /(L \cap R)} \text { as } \lambda \uparrow\right.
\end{aligned}
$$

Therefore

$$
\|a+(L \cap R)\|_{L /(L \cap R)} \leq M\|a+R\|_{L+R / R} \quad(a \in L),
$$

as desired.

The result in $\mathrm{C}^{*}$-algebras follows because closed left ideals in $\mathrm{C}^{*}$-algebras necessarily have bounded right approximate identities, with $M=1$ ([3], 1.7.3).

Later in the paper, we shall produce counterexamples to show that none of the obvious weakenings of Rudin's hypotheses is viable. We have one more positive result to present here; it applies to the special case of uniform algebras. Again, we shall show later that its hypotheses cannot be weakened.

Theorem 2.5. Let $A$ be a uniform algebra and let $I, J$ be closed ideals of $A$ such that $I \cap J=\{0\}$. Then $I+J$ is closed.

Proof. Let $X$ be the character space of $A$. Let

$$
E=h(I):=\{t \in X: f(t)=0 \quad(f \in I)\}
$$

and $F=h(J)$. Then $I \subseteq k(E)$ and $J \subseteq k(F)$. The fact that $I \cap J=\{0\}$ implies that $E \cup F=X$ (for if $t \in X \backslash(E \cup F$ ), then there exist $f \in I, g \in J$ such that $f(t) \neq 0$ and $g(t) \neq 0$; then $f g(t) \neq 0$, but $f g \in I \cap J=\{0\})$.

Now suppose $\left(f_{n}\right) \subseteq I,\left(g_{n}\right) \subseteq J$ are sequences such that $f_{n}+g_{n} \rightarrow h$ in $A$. Then $f_{n} \mid E=0$ and $g_{n} \mid F=0$, for all $n$. Hence

$$
\left\|f_{n}-h\right\|_{F}=\left\|f_{n}+g_{n}-h\right\|_{F} \rightarrow 0 \text { as } n \rightarrow \infty
$$

and

$$
\left\|f_{n}-0\right\|_{E}=0 \quad(n \in \mathbb{N})
$$

Therefore $\left(f_{n}\right)$ is uniformly Cauchy on $E \cup F=X$; i.e. $\left(f_{n}\right)$ is Cauchy in $A$. Therefore, $\left(f_{n}\right)$ converges in $A$, and its limit is its pointwise limit:

$$
h_{1}(t)= \begin{cases}0 & (t \in E) \\ h(t) & (t \in F) .\end{cases}
$$

Thus $h_{1} \in A$; in fact, since $I$ is closed, $h_{1} \in I$. Likewise, $g_{n} \rightarrow h_{2} \in J$ where

$$
h_{2}(t)= \begin{cases}h(t) & (t \in E) \\ 0 & (t \in F) .\end{cases}
$$

Therefore, $f_{n}+g_{n} \rightarrow h_{1}+h_{2} \in I+J$. Thus $I+J$ is closed. 


\section{Degenerate EXAmples}

It is easy to construct examples of closed subspaces of a Banach space whose sum is not closed. Indeed we have

Theorem 3.1 (Rudin [7], Proposition 4.8). Every infinite-dimensional Banach space $X$ contains closed subspaces $Y$ and $Z$, with $Y \cap Z=\{0\}$, such that $Y+Z$ is not closed.

To produce a non-closed sum of closed ideals, it is only necessary to equip an infinite-dimensional Banach space $X$ with a zero multiplication (every product of two elements is zero) to turn these subspaces $Y, Z$ into ideals. The following two questions are designed to avoid this degenerate example, but are nevertheless answered negatively using the same type of construction.

Question 3.2. Let $A$ be a semisimple Banach algebra and let $I, J$ be closed ideals of $A$. Is $I+J$ necessarily closed in $A$ ?

This easily falls to the observation that every subspace of $A$ containing $A^{2}$ is necessarily an ideal. Therefore, if $A$ is a semisimple Banach algebra such that $\overline{A^{2}}$ is of infinite codimension, two closed subspaces of $A / \overline{A^{2}}$ with a non-closed sum will lift to closed ideals of $A$ with a non-closed sum.

Question 3.3. Let $A$ be a Banach algebra with Jacobson radical $R$ and let $I$ be a closed ideal of $A$; is $R+I$ necessarily closed in $A$ ?

In this question, one of the ideals is fixed, so we need an explicit construction to give a counterexample.

Example 3.4. The answer to Question 3.3 is negative: the sum of the radical and a closed ideal need not be closed.

Let $\left(\xi_{i}\right)_{i=1}^{\infty}$ be a sequence of distinct points in $(0,1]$ with $\xi_{i} \rightarrow 0$. Let

$$
B=\left\{f \in C^{1}[0,1]: f\left(\xi_{i}\right)=0 \quad(i=1,2,3, \ldots)\right\},
$$

where the algebra $C^{1}[0,1]$ of all continuously differentiable functions on $[0,1]$ has the usual norm

$$
\|f\|=\sup _{0 \leq t \leq 1}|f(t)|+\sup _{0 \leq t \leq 1}\left|f^{\prime}(t)\right|
$$

and pointwise multiplication. If $f \in B$, then $f^{\prime}(0)=0$, and so $f^{\prime}\left(\xi_{i}\right) \rightarrow 0$.

Let $R$ be the radical Banach algebra consisting of the sequence space $\ell^{1}(\mathbb{N})$ with zero multiplication. Then $R$ is the radical of the Banach algebra $A=B \oplus R$. Let

$$
I=\left\{(f, x) \in A: x(i)=f^{\prime}\left(\xi_{i}\right) \quad(i=1,2,3, \ldots)\right\} .
$$

Then $I$ is a closed ideal of $A$. Now

$$
R+I=R+\left\{f \in B:\left(f^{\prime}\left(\xi_{i}\right)\right) \in \ell^{1}\right\} \neq A .
$$

However, it is easy to see that any element of $B$ can be approximated in the $C^{1}$ norm by elements $f \in B$ with $\left(f^{\prime}\left(\xi_{i}\right)\right) \in c_{00} \subseteq \ell^{1}$; so $\overline{R+I}=A$.

Remark 3.5. If $I, J$ are closed ideals with a non-closed sum in a Banach algebra $A$, then they are also closed ideals with a non-closed sum in the algebra obtained by adjoining an identity to $A$ in the usual way, so the absence of an identity in the algebra $A$ in the above examples is unimportant. 


\section{An example in a Banach function algebra}

The zero multiplication algebra is still the basis for our examples. To try to get away from it we ask the following question.

Question 4.1. Let $A$ be a Banach algebra and let $I, J$ be closed two-sided ideals of $A$ such that $A / I, A / J$ (and therefore $A /(I \cap J)$ ) are all semisimple. Is $I+J$ necessarily closed in $A$ ?

Another question, raised by Rudin's theorem, is:

Question 4.2. Let $A$ be a commutative Banach algebra and let $I, J$ be closed twosided ideals of $A$ which have unbounded approximate identities. Is $I+J$ necessarily closed in $A$ ?

A third question, arising from Theorem 2.5, is:

Question 4.3. Does Theorem 2.5 - that if $I, J$ are closed ideals of a uniform algebra $A$ such that $I \cap J=\{0\}$, then $I+J$ is closed - extend from uniform algebras to general semisimple commutative Banach algebras?

Rudin (7], Theorem 4.9) gives an example which answers Question 4.1 negatively in the disc algebra. A full description of when a sum of closed ideals in the disc algebra is closed is provided in [8].

Here we shall give negative solutions to all three of the above questions using an algebra of differentiable functions on the unit interval.

Theorem 4.4. There is a commutative semisimple Banach algebra with closed ideals $I, J$, each of which has a sequential unbounded approximate identity, such that $A / I$ and $A / J$ and $A /(I \cap J)$ are semisimple and $I+J$ is not closed.

Proof. Let $A=\left\{f \in C^{1}[0,1]: f(0)=0\right\}$. Then $A$ is a commutative semisimple Banach algebra. Let $E, F \subseteq(0,1]$,

$$
E=\bigcup_{i=1}^{\infty}\left[a_{i}, b_{i}\right], \quad F=\bigcup_{i=1}^{\infty}\left[c_{i}, d_{i}\right],
$$

where

$$
b_{1}>a_{1}>d_{1}>c_{1}>b_{2}>a_{2}>d_{2}>c_{2}>b_{3}>\cdots \rightarrow 0 .
$$

Further, we choose these points so that

$$
a_{i}-d_{i}=\gamma_{i}
$$

decays at a suitably fast rate, to be specified later.

Let $I=k(E):=\{f \in A: f \mid E=0\}$, and $J=k(F)$. Then $A / I$ and $A / J$ are semisimple.

Now consider $I+J$. Let $f=g+h$ with $g \in I, h \in J$. Then for each $i$, we have $\left|h\left(a_{i}\right)\right|=\left|f\left(a_{i}\right)\right|$. But $h\left(d_{i}\right)=0$. Therefore there is some $u \in\left(d_{i}, a_{i}\right)$ with

$$
\left|h^{\prime}(u)\right|=\frac{\left|f\left(a_{i}\right)\right|}{\gamma_{i}} .
$$

Therefore

$$
\sup _{i} \frac{\left|f\left(a_{i}\right)\right|}{\gamma_{i}} \leq\|h\|<\infty
$$


i.e. $\left|f\left(a_{i}\right)\right|=O\left(\gamma_{i}\right)$. Thus $I+J \neq A$, provided that the $a_{i}, b_{i}, c_{i}, d_{i}$ are chosen suitably - for example, with $\gamma_{i}=o\left(a_{i}\right)$, so that if $f(t)=t$, then $f \in A \backslash(I+J)$. Let

$$
A_{00}=\{f \in A: f(t)=0 \quad(0 \leq t \leq \delta) \text { for some } \delta>0\} .
$$

Clearly, $A_{00} \subseteq I+J$, and $A_{00}$ is dense in $A$. Therefore $I+J$ is dense in $A$, so $I+J$ is not closed.

Finally, we show that $I, J$ have approximate identities. Given $f_{1}, \ldots, f_{k} \in I$ and $\varepsilon>0$, then $f_{i}^{\prime}(0)=0(1 \leq i \leq k)$, so we can find $m$ such that $\left|f_{i}(t)\right|+\left|f_{i}^{\prime}(t)\right| \leq \varepsilon$ for $1 \leq i \leq k$ and $0 \leq t \leq b_{m+1}$. We define $e \in I$ such that $e(t)=0$ for $0 \leq t \leq b_{m+1}$. In the intervals $\left(b_{j+1}, a_{j}\right)(1 \leq j \leq m)$, we define $e$ so that $\left|e(t) f_{i}(t)-f_{i}(t)\right|+\left|\left(e f_{i}\right)^{\prime}(t)-f_{i}^{\prime}(t)\right|<\varepsilon(1 \leq i \leq k)$. That this can be done follows from the fact that the algebra

$$
\left\{f \in C^{1}[0,1]: f(0)=f(1)=f^{\prime}(0)=f^{\prime}(1)=0\right\}
$$

has an approximate identity. Then $\left\|e f_{i}-f_{i}\right\| \leq \varepsilon(1 \leq i \leq k)$, so $I$ (and likewise $J$ ) has an approximate identity.

Remark 4.5. By taking the quotient of the above algebra by $I \cap J$, we obtain a counterexample for Question 4.3 .

Note, however, that this quotient method does not give us a counterexample to Theorem [2.5] starting from Rudin's example in the disc algebra, because the quotient $A /(I \cap J)$ is not a uniform algebra.

As a curiosity, Example 4.4 provides another route to a uniform algebra counterexample for Question 4.1 .

Corollary 4.6. There is a uniform algebra $A^{\prime}$ with closed ideals $I^{\prime}, J^{\prime}$ such that $A^{\prime} / I^{\prime}$ and $A^{\prime} / J^{\prime}$ are semisimple and $I^{\prime}+J^{\prime}$ is not closed.

Proof. Continuing the notation in the proof of Example 4.4, we note that, by [2, Theorem 4.1, the algebra $C^{1}[0,1]$ is a "Q-algebra", and hence the same is true of the subalgebra $A$; that is, $A$ is isomorphic to a quotient of some uniform algebra $A^{\prime}$. It follows immediately that Example 4.4 lifts to the desired example in $A^{\prime}$.

Remark 4.7. One might observe that uniform algebras are precisely closed subalgebras of commutative $\mathrm{C}^{*}$-algebras and ask whether Theorem 2.5 holds in closed subalgebras of non-commutative $\mathrm{C}^{*}$-algebras. Here again, Q-algebra theory answers the question, since a theorem of Cole and Wermer tells us that every Qalgebra is isomorphic to some closed subalgebra of a $\mathrm{C}^{*}$-algebra. The example of Remark 4.5 is a Q-algebra: it is therefore a (commutative) closed subalgebra of a (non-commutative) $\mathrm{C}^{*}$-algebra and an algebra in which Theorem 2.5 fails.

Remark 4.8. One might consider simplifying Example 4.4 by making $E$ and $F$ from sequences of points rather than sequences of non-trivial closed intervals. However, neither in this simplified example nor in the example of Rudin in the disk algebra do the ideals have approximate identities.

\section{Counterexamples With One-Sided Approximate identities}

Rudin ([7], Theorem 4.7) shows that it is not true that either the sum of two closed right ideals or the sum of two closed left ideals is necessarily closed; counterexamples exist in $\mathcal{B}(X)$ for any infinite-dimensional Banach space $X$. Taking $X$ 
to be Hilbert space, the right ideals concerned have bounded left approximate identities; in fact they have left identities, and the left ideals have right identities.

We shall now show that the sum of a closed left ideal and a closed right ideal, one or both having bounded one-sided approximate identities, can fail to be closed when the approximate identities are on the wrong side. Again, our examples use one-sided identities.

Theorem 5.1. 1) There is a Banach algebra A with a closed (two-sided) ideal I which has a left identity and a closed right ideal $J$ such that $I+J$ is not closed.

2) There is a Banach algebra $B$ with a closed left ideal $L$ which has a left identity and a closed right ideal $R$ which has a right identity, such that $L+R$ is not closed.

Proof. Let $H$ be a Hilbert space, and let $K, J$ be closed subspaces of $H$ such that $J+K$ is not closed.

For 1), let $A=H \oplus \mathbb{C} e$, with norm

$$
\|x+\lambda e\|=\|x\|+|\lambda| \quad(x \in H, \lambda \in \mathbb{C}) .
$$

Let $P: H \rightarrow H$ be the orthogonal projection onto $K$. We give $A$ the multiplication defined by

$$
(x+\lambda e)(y+\mu e)=\lambda P(y)+\lambda \mu e \quad(x, y \in H, \lambda, \mu \in \mathbb{C}) .
$$

It is easily checked that $A$ is a Banach algebra, and that $J$ is a right ideal (since $H A=\{0\})$. We define $I=K \oplus \mathbb{C} e$. Then $I=A^{2}$ is an ideal. Clearly $I$ and $J$ are closed, but $I+J=K+J+\mathbb{C} e$ is not. By construction, $e$ is a left identity for $I$.

For 2), let $B=H \oplus \mathbb{C} e \oplus \mathbb{C} f$, with norm

$$
\|x+\lambda e+\mu f\|=\|x\|+|\lambda|+|\mu| \quad(x \in H, \lambda, \mu \in \mathbb{C}) .
$$

We define the multiplication of $B$ by requiring $\{0\}=H^{2}=H e=f H$, ef $=0$, $e^{2}=e, f^{2}=f$ and $e x=x f=x$ for all $x \in H$. Let $L=K \oplus \mathbb{C} e, R=J \oplus \mathbb{C} f$. Then $L$ is a closed left ideal with left identity $e$ and $R$ is a closed right ideal with right identity $f$, but $L+R=(K+J) \oplus \mathbb{C} e \oplus \mathbb{C} f$ is not closed in $B$. (Notice that the fact that $e f=0$ prevents $e$ from being a left identity for $R$ and $f$ from being a right identity for $L$; the fact that $e x=x \notin L$ for $x \in H \backslash K$ prevents $L$ from being a right ideal, and $x f=x \notin R$ for $x \in H \backslash J$ prevents $R$ from being a left ideal.)

\section{REFERENCES}

1. F. Combes, Sur les faces d'une $C^{*}$-algèbre Bull. Sci. Math., 93 (1969), 37-62. MR 42:856

2. A. M. Davie, Quotient algebras of uniform algebras, J. London Math. Soc. (2), 7 (1973), 31-40. MR 48:2779

3. J. Dixmier, Les $C^{*}$-algèbres et leurs représentations, Gauthier-Villars, Paris, 1964. MR 30:1404

4. P. G. Dixon, Topologically irreducible representations and radicals in Banach algebras, Proc. London Math. Soc. (3), 74 (1997), 174-200. MR 30:46066

5. Robert S. Doran and Josef Wichmann, Approximate Identities and Factorization in Banach Modules, Springer, Lecture Notes in Math., 768, Berlin, 1979. MR 83e:46044

6. E. Kirchberg, On restricted perturbations in inverse images and a description of normalizer algebras in $C^{*}$-algebras, J. Functional Analysis, 129 (1995), 1-34. MR 95m:46094a

7. W. Rudin, Spaces of type $H^{\infty}+C$, Ann. Inst. Fourier, Grenoble, 25 (1975), 99-125. MR 57:13692, errata MR 57:7134 
8. D. A. Stegenga, Ideals in the disk algebra, J. Functional Analysis, 25 (1977), 335-337. MR 58:2307

9. Simon Wassermann, Exact $C^{*}$-algebras and related topics, Research Institute of Mathematics, Global Analysis Research Center, Seoul National University, Lecture Note Series, 19, 1994. MR 95b:46081

Department of Pure Mathematics, University of Sheffield, Sheffield, S3 7RH, EngLAND

E-mail address: P.Dixon@sheffield.ac.uk

$U R L:$ http://www. shef.ac.uk/ ppm1pgd 